\title{
Brane oscillations and the cosmic coincidence problem
}

\author{
A.L. Maroto \\ Departamento de Física Teórica, Universidad Complutense de Madrid, 28040 Madrid, Spain
}

(Dated: November 20, 2018)

\begin{abstract}
We show that, under general assumptions, in six-dimensional brane-world models with compactified large extra dimensions, the energy density of brane oscillations scales as that of cold dark matter and its present value is compatible with observations. Such value is obtained from the only dimensional scale in the theory, namely, the fundamental scale of gravity in six dimensions $M_{6} \sim 1 \mathrm{TeV}$, without any fine-tuning or the introduction of additional mass scales apart from the large size of the extra dimensions. It has been suggested that the same kind of models could provide also the correct magnitude of the cosmological constant. This observation can be relevant for the resolution of the cosmic coincidence problem in the brane-world scenario.
\end{abstract}

PACS numbers: 95.35.+d, 11.25.-w, 11.10.Kk

\section{INTRODUCTION}

Recent cosmological observations [1] seem to favour a critical or nearly critical universe $\left(\Omega_{t o t} \simeq 1\right)$ with an important fraction of cold dark matter $\left(\Omega_{D M} \simeq 0.23\right)$ and dominated by dark energy or a cosmological constant ( $\Omega_{\Lambda} \simeq 0.73$ ), whereas ordinary baryons only account for a small fraction of the total energy density $\left(\Omega_{B} \simeq 0.04\right)$. Since the time evolution of each density parameter is different, the fact that they have a comparable magnitude today suggests that, either we are living a sort of cosmic coincidence, without any deeper explanation, or there is a strong relationship among the origin and evolution of the three types of densities.

Concerning the origin of the dark energy, the introduction of a cosmological constant could appear as the simplest ad hoc solution, however the required value $\rho_{\Lambda} \simeq\left(2 \cdot 10^{-3} \mathrm{eV}\right)^{4}$ is much smaller than any natural scale arising in particle physics models (apart from the neutrino mass) and therefore fine-tuning or the introduction of new physics seems unavoidable. A more satisfactory approach, as commented before, would be to find a link between the evolution of dark energy and matter. This is the main idea behind the so called quintessence models 2], in which a light scalar field is rolling down an exponential potential. It can be seen that the attractor solution of this system mimics the evolution of the dominant component of the universe (tracker solution). However, the potential parameters still require to be fine-tuned in order to get quintessence domination today. More recently, tracker behaviours have also been found in the so called k-essence models [3] which are based on scalar fields with non-canonical kinetic terms. They are claimed to solve the coincidence problem without fine-tuning, (see however [4]).

On the other hand, the most popular solutions to the dark matter problem (see [5] and references therein), i.e. the existence of an important thermal relic abundance of weakly interacting massive particles, such as the neu- tralino, or a non-thermal background of axions, seem to be unrelated to the cosmological constant value. Another possible link between them was suggested in [6] There, it was shown that the existence of an ultra-light pseudo-Nambu-Goldstone boson with a mass of the order of the Hubble parameter today $M \sim H_{0} \sim 10^{-33}$ $\mathrm{eV}$ and a spontaneous symmetry breaking scale $v \sim \bar{M}_{P}$, where $\bar{M}_{P}=M_{P} / \sqrt{8 \pi}$ is the reduced Planck mass, could explain the current amount of dark matter/energy. In fact, similarly to axionic dark matter, the energy density of the oscillations of the pseudo-GB fields scales as cold dark matter, and since the initial amplitude of such oscillations is set by the $v$ scale, its present density is naturally of the correct order of mangitude $\rho_{D M} \sim M^{2} \bar{M}_{P}^{2}$ provided the oscillations have not been damped too much by the present time. This is consistent with the fact that, in this scenario, oscillations start only when $3 H \lesssim M$, otherwise the friction term due to the universe expansion freezes the scalar field to its initial value. This condition can be written, using the Friedmann equation as $H^{2} \sim \rho_{\text {tot }} / \bar{M}_{P}^{2} \sim M^{2}$, which implies $\rho_{\text {tot }} \sim M^{2} \bar{M}_{P}^{2} \sim \rho_{D M}$, as commented before. Notice also that if today $3 H \gtrsim M$, the pseudo-GB energy density would contribute as a cosmological constant. The main difficulty in this proposal is precisely to find a pseudo-GB candidate whose dynamics is given by two such vastly different scales ( $M$ and $v$ ).

More recently, the coincidence problem has been considered in the context of large extra dimensions models. Thus for example, the radion field which determines the size of the extra dimension has been proposed as a quintessence candidate in 7]. Also radion oscillations have been shown to potentially contribute to the present acceleration of the universe or as a dark matter energy density [8]. A very interesting observation was given in [9, 10] and it is the fact that in the Arkani-Hamed, Dimopoulos, Dvali (ADD) brane-world model [11], with a fundamental gravity scale $M_{D} \sim 1 \mathrm{TeV}$, the size of the compactified extra dimensions is comparable to the dark 
energy scale $R_{B}^{-1} \sim 10^{-3} \mathrm{eV}$, provided the total number of dimensions is $D=6$. Thus, the vacuum energy density due to loops of light fields propagating in the bulk space is $\mathcal{O}\left(R_{B}^{-D}\right)$ 12. Integrating the extra-space volume, the corresponding contribution to the four-dimensional cosmological constant has precisely the correct order of magnitude $\rho_{\Lambda} \sim R_{B}^{-4}$. This fact has lead to the construction of different dark-energy models with dynamical moduli fields [13, 14]. An additional interesting property of sixdimensional models is the fact that the brane tension does not contribute to the brane cosmological constant, its only effect is the generation of a deficit angle in the bulk metric. This observation suggests that the amount of fine-tuning needed to solve the cosmological constant problem could be reduced in these models [10, 15].

In this paper we will explore the dark matter problem in six-dimensional brane-world models, considering the brane as a dynamical object which can move and fluctuate along the extra dimensions. The fields parametrizing the position of the brane in the extra space are known as branons, and they have been shown to be natural candidates to dark matter, both as thermal [16] and nonthermal 17] relics. In addition, such fields can be understood as the Goldstone bosons corresponding to the spontaneous breaking of the traslational isometries in the extra space [18, 19]. When such isometries are explicitly broken, due to the curvature of the extra dimensions, such fields are no longer massless and therefore they could play the role of the pseudo-GB fields in the reference [6]. In this work, we will show that the mass $M$ and the spontaneous breaking scale $v$ are naturally of the correct order of magnitude, provided the bulk vacuum energy is $\mathcal{O}\left(R_{B}^{-6}\right)$, i.e., comparable to that generated by quantum effects in the compact dimensions.

The paper is organized as follows. In section 2, we review the main properties of branon fields and relate their mass to the curvature of the bulk space. In section 3 , we study the possibility that dark matter could be in the form of brane oscillations. Section 4 is devoted to an explicit example based on the so called anti-de Sitter $\left(\mathrm{AdS}_{6}\right)$ soliton solution, and finally in section 5 we discuss some difficulties in the builiding of realistic models and give the main conlusions of the work.

\section{BRANE FLUCTUATIONS}

Let us consider 20, 21] our four-dimensional spacetime $M_{4}$ to be embedded in a 6 -dimensional bulk space which for simplicity will be assumed to be of the form $M_{6}=M_{4} \times B$, where $B$ is a given compact manifold. The coordinates parametrizing the points in $M_{6}$ will be denoted by $\left(x^{\mu}, y^{m}\right)$, where the different indices run as $\mu=0,1,2,3$ and $m=4,5$.

The bulk space $M_{6}$ is endowed with a metric tensor that we will denote by $G_{M N}$, with signature $(+,-,-\ldots-,-)$. For simplicity, we will consider the following ansatz:

$$
G_{M N}=\left(\begin{array}{cc}
\tilde{g}_{\mu \nu}(x, y) & 0 \\
0 & -g_{m n}^{\prime}(y)
\end{array}\right),
$$

where

$$
\tilde{g}_{\mu \nu}(x, y)=g_{\mu \nu}(x)\left(1+\sigma\left(y^{2}\right)\right),
$$

with $\sigma(0)=0$ and $y^{2}=\left(y^{4}\right)^{2}+\left(y^{5}\right)^{2}$. We have chosen $y^{m}$ as normal and geodesic coordinates at least with respect to the $y=0$ point, so that we can write $g_{m n}^{\prime}=\delta_{m n}+$ $\mathcal{O}\left(y^{2}\right)$. In the models in which we will be interested, the warp factor in the metric will be almost irrelevant since, as will we see below, $\sigma\left(y^{2}\right) \ll 1$. In such a case, the usual relation of the ADD models between the four and D-dimensional gravity scales $M_{P}^{2}=V_{B} M_{D}^{D-2}$ still holds, so that if we fix $M_{D} \sim 1 \mathrm{TeV}$, the size of the extra dimensions should be $R_{B}^{-1} \sim 10^{-3} \mathrm{eV}$.

We will work in the probe-brane approximation, so that we assume the 3-brane is moving in the background metric given by (1) which is not perturbed by its presence. The position of the brane in the bulk can be parametrized as $Y^{M}=\left(x^{\mu}, Y^{m}(x)\right)$, where we have chosen the bulk coordinates so that the first four are identified with the space-time brane coordinates $x^{\mu}$. We assume for simplicity that the ground state of the brane corresponds to $Y^{m}(x)=Y_{0}^{m}=0$.

When $\sigma\left(y^{2}\right) \equiv 0$ we will assume that the $M_{6}$ isometry group can be written as $G\left(M_{6}\right)=G\left(M_{4}\right) \times G(B)$. The presence of the brane will break spontaneously all the $B$ isometries except for those that leave the point $Y_{0}$ unchanged. In other words the group $G(B)$ is spontaneously broken down to $H\left(Y_{0}\right)$, where $H\left(Y_{0}\right)$ denotes the isotropy group of the point $Y_{0}$. The excitations of the brane along the (broken) Killing fields directions of $B$ correspond to the zero modes and they are parametrized by the GB fields $\pi^{\alpha}(x), \alpha=4,5$ that can be understood as coordinates on the coset manifold $K=G(B) / H\left(Y_{0}\right)$. Let us assume that the number of GB fields equals the dimension of $B$. In that case we can choose the coordinates on $B$ and $K$ so that

$$
\pi^{\alpha}(x)=\frac{v}{R_{B}} \delta_{m}^{\alpha} Y^{m}(x)=f^{2} \delta_{m}^{\alpha} Y^{m}(x),
$$

where

$$
v=f^{2} R_{B},
$$

is the size of $K, R_{B}$ is the radius of $B$ and $f$ is the brane tension scale.

In the general case, $\sigma\left(y^{2}\right) \neq 0$, the $G(B)$ isometries will be both spontaneous and explicitly broken. Thus, expanding around $y=0$, the induced metric on the brane is written in terms of branon fields as:

$$
\begin{aligned}
& g_{\mu \nu}(x, \pi)=\tilde{g}_{\mu \nu}(x, Y)-\frac{1}{f^{4}} \partial_{\mu} \pi^{\alpha} \partial_{\nu} \pi^{\alpha} \\
& =g_{\mu \nu}(x)\left(1+\frac{M^{2} \pi^{2}}{4 f^{4}}\right)-\frac{1}{f^{4}} \partial_{\mu} \pi^{\alpha} \partial_{\nu} \pi^{\alpha}+\mathcal{O}\left(\pi^{4}\right)
\end{aligned}
$$


with $\pi^{2}=\left(\pi^{4}\right)^{2}+\left(\pi^{5}\right)^{2}$ and $M^{2}=4 \sigma^{\prime}(0)$ where the prime denotes derivative with respect to $y^{2}$.

Introducing this expansion into the Nambu-Goto action for the brane we get, up to terms quadratic in the $\pi$ fields:

$$
\begin{aligned}
S_{B r} & =-f^{4} \int_{M_{4}} d^{4} x \sqrt{g(x, \pi(x))}=-\int_{M_{4}} d^{4} x \sqrt{g(x)} f^{4} \\
& +\int_{M_{4}} d^{4} x \sqrt{g(x)} \frac{1}{2}\left(g^{\mu \nu} \partial_{\mu} \pi^{\alpha} \partial_{\nu} \pi^{\alpha}-M^{2} \pi^{2}\right) .
\end{aligned}
$$

Notice that the warp factor is responsible for the generation of a mass term for the branon field. Thus, expressing the bulk Ricci tensor in terms of its lower-dimensional counterparts, it is possible to relate the branon mass to the bulk curvature as:

$$
M^{2}=-\left.\frac{1}{2}\left(R^{\prime}+R_{m}^{m}\right)\right|_{y=0},
$$

where $R^{\prime}$ is the curvature scalar corresponding to the $g_{m n}^{\prime}$ metric and $R_{m}^{m}=-g_{m n}^{\prime} R^{m n}$, with $R_{m n}$ the internal components of the Ricci tensor corresponding to the bulk metric. Notice that this expression holds regardless the particular form of the $g_{m n}^{\prime}$ metric, provided the bulk metric satisfies the conditions imposed above.

Branons also interact with the Standard Model (SM) particles through their energy momentum tensor. Again the lowest order term was obtained in [19, 20]:

$$
\mathcal{L}_{B r-S M}=\frac{1}{8 f^{4}}\left(4 \partial_{\mu} \pi^{\alpha} \partial_{\nu} \pi^{\alpha}-M^{2} \pi^{2} g_{\mu \nu}+\ldots\right) T_{S M}^{\mu \nu}
$$

where the dots stand for higher order terms in $\pi$ fields. We see that the branon-SM interactions are controlled by the brane tension scale $f$.

\section{DARK MATTER FROM BRANE OSCILLATIONS}

In 16. it was shown that branons could be produced by the freeze-out mechanism in an expanding universe through their couplings to the SM particles in (8). Thus if branons decoupled early enough, their cosmological abundance today can be relevant and they could account for the dark matter of the universe. However, apart from their thermal production, it is also possible to produce branons non-thermally, very much in the same way as in the misalignment mechanism for axions [17]. In such a case, due to the mass term of branon fields, the energy density stored in the coherent oscillations of the brane around the potential minimun could account for the observed dark matter, provided the mass $M$ and the oscillations amplitude have the correct magnitude.

Let us briefly review that mechanism. If the maximum temperature reached in the universe was smaller than the branon freeze-out temperature $T_{R H} \ll T_{f}$, but larger compared to the explicit symmetry breaking scale $T_{R H} \gg \lambda$ with $\lambda=(M v)^{1 / 2}$, then brane fluctuations were initially essentially massless and decoupled from the rest of matter fields. In this case, there is no reason to expect that the position in the extra dimension $Y_{c}$ at which the brane is created should coincide with the minimum of the branon potential $(Y=0)$. In general we expect $Y_{c} \sim \mathcal{O}\left(R_{B}\right)$, i.e. $\pi_{c} \sim v$ within a region of size $H^{-1}$ [22]. The evolution of the branon field is then simply that of a scalar field in an expanding universe. Thus, while $H \gg M$, the field remains frozen in its initial position $\pi=\pi_{c}$. Below the temperature $T_{i}$ for which $3 H\left(T_{i}\right) \simeq M$, the branon field oscillates around the minimum. These oscillations correspond to a zero-momentum branon condensate, its energy density behaving like nonrelativistic matter 23].

Let us define $\Gamma(T)$ as the total branon annihilation rate including annihilations into SM particles and $4 \pi \rightarrow 2 \pi$ processes. In the case in which $H(T)>\Gamma(T)$ throughout the history of the universe, the branon condensate energy density essentially is not reduced by particle production, but only diluted by the Hubble expansion. In such a case the coherent brane oscillation can survive until present. The specific conditions for this to happen were obtained in 17 and they are summarized in the equation: $T_{i} \simeq\left(M M_{P}\right)^{1 / 2}<T_{R H}<T_{f}$. For light branons, a good estimation for the freeze-out temperature was obtained in 16, 24]: $\log \left(T_{f} / \mathrm{GeV}\right) \simeq(8 / 7) \log (f / \mathrm{GeV})-3.2$. Notice that in the case in which we will be mainly interested, with only one fundamental scale, i.e. $f \sim M_{D} \sim$ $\mathrm{TeV}$, the freeze-out temperature is $T_{f} \sim \mathrm{GeV}$, and therefore the previous condition is compatible with a reheating temperature above the nucleosynthesis temperature $T_{R H}>T_{B B N} \sim \mathrm{MeV}$. On the other hand, in order for the above interval to exist, the branon mass should satisfy $M<10^{-10} \mathrm{eV}$.

When these conditions are satisfied, we can calculate the energy density which is stored today in the form of brane oscillations. Assuming that $M$ does not depend on the temperature, it is given by [17, 22]:

$$
\Omega_{B r} h^{2} \simeq \frac{2.5 N v^{2} M T_{0}^{3}}{M_{P} T_{i} \rho_{0}} \simeq \frac{6.5 \cdot 10^{-20} N}{\mathrm{GeV}^{5 / 2}} f^{4} R_{B}^{2} M^{1 / 2},
$$

where $T_{0}$ and $\rho_{0}$ are the photon temperature and critical density today respectively, and $N$ is the number of branon fields. We see that in order for the branon condensate to be responsible for the dark matter abundance $\Omega_{B r} h^{2} \simeq 0.1$, the branon mass $M$ should be in the range $M=10^{-27}-10^{-35} \mathrm{eV}$, for $f=1-10 \mathrm{TeV}$ and $R_{B}^{-1}=10^{-3} \mathrm{eV}$. Notice also that in this range $v=f^{2} R_{B} \sim \bar{M}_{P}$. Since $H_{0} \simeq 10^{-33} \mathrm{eV}$, the brane could have started oscillating before the present time if $M>H_{0}$ (its energy density scaling as dark matter) or 
still be frozen at its initial point if $M<H_{0}$ (cosmological constant). In order to calculate $M$ we need to specify the bulk energy-momentum tensor. In the following we will consider the simplest non-trivial model in which the bulk space only contains a cosmological constant.

\section{AN EXAMPLE: ADS $_{6}$ SOLITON}

Let us consider the solutions of Einstein equations in six-dimensional space-time with a (negative) cosmological constant $\Lambda_{6}$. When the extra space has azimuthal symmetry and the metric depends only on the radial coordinate $\rho$ with a periodic angular coordinate $\theta \in[0,2 \pi)$, a simple solution is given by [25, 26]:

$$
d s^{2}=M^{2}(\rho) \eta_{\mu \nu} d x^{\mu} d x^{\nu}-d \rho^{2}-L^{2}(\rho) d \theta^{2},
$$

where

$$
M(\rho)=\cosh ^{2 / 5}(k \rho) ; \quad L(\rho)=\frac{\sinh (k \rho)}{k \cosh ^{3 / 5}(k \rho)},
$$

with:

$$
k=\sqrt{-\frac{5 \Lambda_{6}}{8 M_{6}^{4}}} .
$$

Notice that the metric is normalized so that for $k \rho \ll 1$, we recover the Minkowskian form. Notice that we have assumed that the presence of the brane has no effect on the bulk metric. However, even if we include the jump conditions at the brane position, it can be seen that the only consequence would be the introduction of a deficit angle in the $\theta$ coordinate, which is related to the brane tension 26]. In addition, in order to compactify the extra dimensions, it has been shown [10, 26] that it is possible to truncate the extra space by introducing a 4-brane at a finite distance $\rho=R_{B}$ with an anisotropic energymomentum tensor.

It is possible to write the above metric in quasiMinkowskian extra-coordinates $y^{4}=\rho \cos (\theta), y^{5}=$ $\rho \sin (\theta)$. Expanding around $y=0$ we find for the nonvanishing metric components in the new coordinates:

$$
\begin{aligned}
G_{\mu \nu} & =\left(1+\frac{2}{5} k^{2} y^{2}+\ldots\right) \eta_{\mu \nu} \\
G_{44} & =-\left(1-\frac{4}{3} k^{2}\left(y^{5}\right)^{2}+\ldots\right) \\
G_{55} & =-\left(1-\frac{4}{3} k^{2}\left(y^{4}\right)^{2}+\ldots\right)
\end{aligned}
$$

Notice also that the extra-coordinates curves with either $y^{4}=0$ or $y^{5}=0$ are normal and geodesic with respect to the origin and therefore define properly normalized branon fields, so that we can obtain the corresponding mass as:

$$
M^{2}=4 \sigma^{\prime}(0)=\frac{8 k^{2}}{5}=-\frac{\Lambda_{6}}{M_{6}^{4}} .
$$

We see that the branon mass is determined by the bulk cosmological constant. If we had considered instead a de Sitter $\mathrm{dS}_{6}$ background solution, $M^{2}$ would be negative and the system unstable.

In order to obtain $M$ we need to make some assumption about the value of $\Lambda_{6}$. Thus, in principle, there would be two natural scales for the bulk cosmological constant namely, the $\mathrm{TeV}$ scale or $R_{B}^{-1}$. Indeed, as commented before if its origin is related to quantum loop effects in the bulk then it would be possible to set $\Lambda_{6} \sim R_{B}^{-6}$. In such a case the branon mass is: $M \sim 1 /\left(M_{6}^{2} R_{B}^{3}\right) \sim 10^{-33} \mathrm{eV}$, and this value could give rise to the correct dark matter fraction for a brane tension scale $f \sim \mathrm{TeV}$, as shown before. Notice also that for this value of $M$, we have $k y \ll 1$ and $\sigma\left(y^{2}\right) \ll 1$ for $y^{2} \leq R_{B}^{2}$ as required. This means that it is possible to neglect $k^{4} y^{4}$ and higher-order terms in the branon potential expansion coming from (13).

On the other hand, if the bulk cosmological constant is of order $\Lambda_{6} \sim \mathrm{TeV}^{6}$, the branon mass would be also $M \sim \mathrm{TeV}$. In such a case, non-thermal production is not possible, and the $\sigma \ll 1$ condition is not satisfied for large extra dimension, accordingly an exponential warp factor would be present in the bulk metric.

Notice that in the former example, starting from a fundamental scale $M_{6} \sim f \sim \mathrm{TeV}$, as suggested by the solution of the gauge hierarchy problem, the four dimensional cosmological constant $\Lambda_{4} \sim R_{B}^{-4}[10$, and the dark matter energy densities would be comparable and with the correct order of magnitude. Thus, in this model, the two problems, i.e. the gauge hierarchy and the cosmic coincidence, are related to a single one, which is the existence of a large size for the extra dimensions.

\section{CONCLUSIONS AND DISCUSSION}

In this paper we have studied the dark matter problem in six-dimensional models with compactified large extra dimensions. In particular, we have shown that if the brane is coherently oscillating in a background metric with an appropriate curvature, the energy density of the oscillations could be seen from the four-dimensional point of view as cold dark matter. In particular, if the background metric is of the $\mathrm{AdS}_{6}$ form with a cosmological constant scale set by the size of the extra dimensions, then the dark matter fraction could be compatible with the value favoured by observations. In such a case, the amplitude of the oscillations $v \sim \bar{M}_{P}$ is set by the only dimensional scales in the problem: $f \sim \mathrm{TeV}$ and $R_{B}$. Notice that this possibility is only present in sixdimensional models. For higher dimensions, the typical values of $R_{B}^{-1}$ and $M$ would be much larger, and the corresponding value of $\Omega_{B r}$ much smaller.

Although the model presented above provides the correct estimation for the dark matter energy density with- 
out including additional mass scales, it is in certain aspects very simplistic. Thus:

1) A flat Minkowskian metric has been assumed on the brane. The possibility of finding more realistic FRW type solutions has been analysed in [26], (see also [27]).

2) We have taken the simplest example in which the bulk only contains a cosmological constant. However quantum effects in the bulk would rather generate an anisotropic Casimir stress tensor. This also poses the problem of the stabilization of the size of the extra dimensions [13, 14].

3) In order to get the correct value for the fourdimensional cosmological constant, we would need a mechanism to protect it against brane or bulk quantum effects. Some proposals include bulk supersymmetry or diffeomorphism invariance [10, 14, 15].

The branon mass $M$ could receive radiative corrections from SM matter loops on the brane through the couplings in (8). However, in the limit of massless branons, the Lagrangian is invariant under the shift symmetry $\pi(x) \rightarrow \pi(x)+C$. This guarantees that polinomial corrections in $\pi$ fields are not generated. So, the only possible branon mass renormalization would come from the $f^{-4} M^{2} \pi^{2} T_{S M \mu}^{\mu}$ term in the Lagrangian. The natural cut-off scale for SM loops in the brane would be $\Lambda \sim M_{D} \sim 1 \mathrm{TeV}$. However, since in the example considered in the previous section we have $f \sim M_{D}$, the renormalized mass would read:

$$
M_{R}^{2}=M^{2}\left(1+\frac{\alpha \Lambda^{4}}{f^{4}}\right)
$$

with $\alpha$ a numerical coefficient of order one or even smaller. This means that the tiny branon mass is only renormalized by an order one factor.

Finally, one could worry about the possibility that such an ultra-light branon field should have been already detected both, as a modification of the Newton law or in collider experiments. However as shown in 28], the fact that branons interact by pairs (see (8)) implies that their contribution to the gravitational potential occurs only at one loop level and their effect is actually unobservable for $f \sim \mathrm{TeV}$, even in the massless limit. In addition, such value for $f$ also ensures that branons cannot be detected in current high-energy collider experiments [20, 29]. However, for brane tensions in that range, their signals could be seen in the next generation of linear [20] or hadronic colliders 30].

Acknowledgements: I would like to thank A. Dobado and J.A.R. Cembranos for useful comments. This work has been partially supported by the DGICYT (Spain) under the project numbers FPA 2000-0956 and BFM2002-01003.

[1] D.N. Spergel and others, Astrophys. J. Suppl. 148, 175 (2003)
[2] B. Ratra and P.J.E. Peebles, Phys. Rev. D37, 3406 (1988); P.J.E. Peebles and B. Ratra, Rev. Mod. Phys. 75, 559-606 (2003); R.R. Caldwell, R. Dave and P.J. Steinhardt, Phys. Rev. Lett. 80, 1582-1585 (1998); I. Zlatev, L-M. Wang and P.J. Steinhardt, Phys. Rev. Lett. 82, 896-899 (1999)

[3] T. Chiba, T. Okabe and M. Yamaguchi, Phys. Rev. D62, 023511 (2000); C. Armendariz-Picon, V. Mukhanov and P.J. Steinhardt, Phys. Rev. Lett. 85, 4438-4441 (2000)

[4] M. Malquarti, E.J. Copeland and A.R. Liddle, Phys. Rev. D68, 02351 (2003)

[5] G. Jungman, M. Kamionkowski and K. Griest, Phys. Rep. 267, 195 (1996); C. Muñoz, hep-ph/0309346

[6] J.A. Frieman, C.T. Hill, A. Stebbins and I. Waga, Phys. Rev. Lett. 75, 2077-2080 (1995)

[7] A. Albrecht, C.P. Burgess, F. Ravndal and C. Skordis, Phys. Rev. D65, 123507 (2002)

[8] L. Perivolaropoulos and C. Sourdis, Phys. Rev. D66, 084018 (2002)

[9] R. Sundrum, Phys. Rev. D59, 085010 (1999)

[10] J.-W. Chen, M.A. Luty and E. Ponton, JHEP 0009, 012 (2000)

[11] N. Arkani-Hamed, S. Dimopoulos and G. Dvali, Phys. Lett. B429, 263 (1998); N. Arkani-Hamed, S. Dimopoulos and G.R. Dvali, Phys. Rev. D59, 086004 (1999); I. Antoniadis, Phys. Lett. B246 377 (1990)

[12] P. Candelas and S. Weinberg, Nucl. Phys. B237, 397 (1984)

[13] M. Pietroni, Phys. Rev. D67, 103523 (2003)

[14] M. Peloso and E. Poppitz Phys. Rev. D68, 125009 (2003)

[15] Y. Aghababaie, C.P. Burgess, S.L. Parameswaran and F. Quevedo, Nucl. Phys. B680, 389-414 (2004)

[16] J.A.R. Cembranos, A. Dobado and A.L. Maroto, Phys. Rev. Lett. 90, 241301 (2003)

[17] A.L. Maroto, Phys. Rev. D69, 043509, (2004)

[18] R. Sundrum, Phys. Rev. D59, 085009 (1999)

[19] A. Dobado and A.L. Maroto, Nucl. Phys. B592, 203 (2001)

[20] J. Alcaraz, J.A.R. Cembranos, A. Dobado and A.L. Maroto, Phys. Rev. D67, 075010 (2003)

[21] J.A.R. Cembranos, A. Dobado and A.L. Maroto, Phys. Rev. D65, 026005 (2002)

[22] J. Preskill, M.B. Wise and F. Wilczek, Phys. Lett. B120, 127 (1983); J.A. Frieman and A.H. Jaffe, Phys. Rev. D45, 2674 (1992)

[23] M.S. Turner, Phys. Rev. D28, 1243 (1983)

[24] J.A.R. Cembranos, A. Dobado and A.L. Maroto, Phys. Rev. D68, 103505 (2003)

[25] F. Leblond, R.C. Myers and D.J. Winters, JHEP 0107, 031 (2001)

[26] J.M. Cline, J. Descheneau, M. Giovannini and J. Vinet, JHEP 0306, 048 (2003)

[27] P. Bostock, R. Gregory, I. Navarro and J. Santiago, hep-th/0311074

[28] T. Kugo and K. Yoshioka, Nucl. Phys. B594, 301 (2001)

[29] P. Creminelli and A. Strumia, Nucl. Phys. B596 125 (2001)

[30] J.A.R. Cembranos, A. Dobado and A.L. Maroto, work in preparation. 